\title{
Análisis del rol de los exámenes de imagen en el Régimen de Garantías Explícitas en salud en Chile
}

Paula Tupper G. ${ }^{1}$, Eugenio Zalaquett . $^{1 *}$, Andrea Fuentealba C. ${ }^{1}$, Juan Pablo Cruz ${ }^{2}{ }^{2}$, Carlos Riquelme $P_{.}{ }^{2}$, Jaime Recasens $T^{3}$

1. Sociedad Chilena de Radiologia, Santiago, Chile.

2. Pontificia Universidad Catolica de Chile, Santiago, Chile.

3. Hospital Dr. Eduardo Pereira Valparaíso, Universidad de Valparaíso. Valparaíso-Chile.

\section{Analysis of the Role of Imaging Exams in the Regime of Explicit Health Guarantees in Chile}

Resumen: Objetivo: Describir la participación de los exámenes de imagen en el Régimen de Garantías Explícitas en Salud en Chile y analizar el papel de los radiólogos en este campo. Materiales y métodos: todas las patologías incluidas en el Programa de garantías explícitas en salud, cuentan con una guía de práctica clínica (GC) y un listado de prestaciones específicas (LP). Ambos fueron analizados respecto a la cantidad y la modalidad de los exámenes de imagen recomendados, la presencia de radiólogos dentro de los paneles de expertos y la concordancia entre las recomendaciones de las guías y los Listados de Prestaciones. Resultados: 60 GC (67\%) y 55 LP (69\%) incluyen pruebas de imagen dentro de sus prestaciones garantizadas. 7 GC (8\%) recomiendan pruebas de imágenes no cubiertas en su listado de prestaciones respectivos y 5 pruebas de imágenes del LP (6\%) no están incluídas en las guías clínicas. La participación de un radiólogo en el panel de expertos se asoció con la ausencia de discrepancias en las pruebas de imagen entre GC y LP ( $p=0,007)$. Discusión: el diagnóstico por imágenes juega un papel importante dentro del Programa de garantías explícitas y se asocia al aumento de los costos de atención médica. Algunos casos de discordancia entre las guías clínicas y los Listados de Prestaciones representan costos económicos y sociales significativos que podrían reducirse al incluir radiólogos en los paneles de expertos, así como optimizar el uso de recursos y reducir la exposición de los pacientes a la radiación ionizante.

Palabras clave: Diagnóstico por imágenes, Guías de práctica clínica, Radiología.

Abstract: Objective: To describe the involvement diagnostic imaging exams in the framework of Regime of Explicit Health Guarantees in Chile and analyze the role of radiologists in this field. Materials and methods: Every pathology included in the Explicit Healthcare Guarantees Program encompasses an expert consensus clinical guideline (CG) and a specific services list (SL). Both of them were analyzed regarding the amount and modality of imaging exams recommended, the presence of radiologists within the expert panels and the concordance between guidelines recommendations and service lists. Results: 60 CG (67\%) and 55 SL (69\%) include imaging tests within their guaranteed services. Seven CG (8\%) recommend medical imaging tests not covered in their respective services list and 5 SL (6\%) reference imaging tests not included in the clinical guidelines. The involvement of a radiologist on the expert panel was associated with the absence of imaging test discrepancies between $C G$ and $S L(p=0.007)$. Discussion: Diagnostic imaging plays an important role within the Explicit Healthcare Guarantees Program and is associated with rising healthcare costs. There are cases of discordance between clinical guidelines and specific services lists that account for significant economic and social costs, which may be reduced by including radiologists on expert panels, optimizing resource use and lowering patients' exposure to ionizing radiation.

Keywords: Diagnostic Imaging, Practice guideline, Radiology.

Zalaquett E., et al. Análisis del rol de los exámenes de imagen en el Régimen de Garantías Explícitas en Salud en Chile Rev Chil Radiol 2019; 25(4): 114-118.

*Correo electrónico: Eugenio Zalaquett / erzalaqu@uc.cl

Trabajo enviado el 31 de octubre de 2019. Aceptado para publicación el 29 de noviembre de 2019. 


\section{Introducción}

El año 2005, en el marco de la ley 19.966 de Chile, se estableció el Régimen de Garantías Explícitas de Salud, las que son relativas a acceso, calidad, oportunidad y protección financiera, contemplando una serie de patologías priorizadas a partir de un algoritmo que consideró criterios de magnitud, trascendencia (AVISA), mortalidad, calidad, preferencia de los usuarios y vulnerabilidad. También se consideraron elementos como carga financiera y suficiencia de la oferta. Basados en esta priorización, entre 2005 y 2006 se definieron 25 problemas de salud, en 2007 éstos aumentaron a 56. El año 2010 se sumaron 13 nuevas patologías con lo que se llegó a 69 problemas de salud GES y para el año 2013 se completó la lista actual de 80 patologías ${ }^{(1,2,3)}$. Las que llegarán a 85 durante el $2019^{(4)}$.

\section{Guías Clínicas GES}

Actualmente son 80 las patologías incluidas en el Régimen de Garantías Explícitas. Por cada patología que se suma, se reúne un equipo de expertos que realiza una revisión bibliográfica exhaustiva del tema y elabora una guía clínica con las recomendaciones correspondientes. Estos documentos son de libre acceso y de carácter público(5).

\section{Listado de Prestaciones Específicas}

El Listado de Prestaciones Específicas es un anexo del Decreto de Garantías Explícitas en Salud que corresponde a la descripción taxativa de las prestaciones unitarias o los grupos de prestaciones determinados en el Arancel de Referencia para cada problema de salud(6). Cuenta con 80 ítems, uno por cada patología (no por cada guía), con sus respectivas prestaciones detalladas.

El uso de los estudios de diagnóstico por imágenes se ha incrementado en el mundo en forma dramática en la última década, aumentando en consecuencia los costos asociados y la exposición a radiaciones ionizantes por causa médica(7). Parte de este aumento está explicado por el positivo papel que ha desempeñado la imagenología en la reformulación del proceso diagnóstico gracias a la existencia de métodos cada vez más certeros, seguros y no invasivos ${ }^{(8)}$.

En Chile, como en el resto del mundo, el acceso a exámenes más certeros y rápidos para llegar a un diagnóstico ha fomentado el aumento en el uso de las imágenes médicas. Entre los años 2012 y 2014 , la imagenología en el sistema de prestadores privados en Chile, incrementó su uso en un $13 \%$, por encima del incremento en precios (4\%), lo que aumentó su costo en un $9 \%$ promedio anual( ${ }^{(9)}$.

El presente trabajo tiene como objetivo analizar la relevancia y el apropiado uso de los exámenes imagenológicos en las Guías Clínicas GES y en el Listado de Prestaciones Específicas.

\section{Materiales y métodos} Fuentes de información

1.a. Guías GES

Para las 80 patologías GES se analizaron todas las guías disponibles. Se incluyó únicamente las versiones más recientes de cada guía. Se consideró sólo el material disponible en la fuente oficial del Ministerio de salud de Chile ${ }^{(10)}$, obviando, por tanto, información adicional como la proveniente de asociaciones de pacientes, sociedades médicas/ científicas o protocolos de prestadores de salud.

\section{1.b. Listado de Prestaciones Específicas}

Dado que las guías clínicas corresponden a un cuerpo de conocimiento estructurado resultado de una revisión de la literatura que presenta recomendaciones, pero no necesariamente garantiza la prestación de los exámenes incluidos, se consideró para el presente estudio el Listado de Prestaciones Específicas buscando todos los exámenes imagenológicos garantizados. Se utilizó la última versión disponible del año 2016 disponible en la fuente oficial del Ministerio de Salud(11).

\section{Datos de interés}

2.a. Exámenes imagenológicos

Se recopiló la cantidad de exámenes imagenológicos recomendados en cada guía GES, el número y modalidad de estudio. Asimismo, para el Listado de Prestaciones se consideró el número y modalidad de estudio por cada patología.

\section{2.b. Presencia de Médico Radiólogo.}

Se revisó el ítem grupo de trabajo de cada guía GES con el objeto de obtener información de que al menos un participante del equipo fuera un Médico Radiólogo.

2.c. Concordancia entre guía GES y Listado de Prestaciones.

Se evaluó si la recomendación de exámenes imagenológicos en la Guía Clínica se garantizaba en el Listado de Prestaciones y, asimismo, si la presencia de exámenes imagenológicos en el Listado de Prestaciones a su vez estaba respaldada por una recomendación en la respectiva Guía GES.

\section{Análisis estadístico}

Se realizó una cuantificación del total de exámenes imagenológicos, luego una categorización por Guía Clínica y por modalidad de estudio.

Se realizó un análisis descriptivo de los exámenes imagenológicos por Guía Clínica GES, expresando dicho resultado como mediana con rango intercuartil.

Se calculó la proporción de guías GES que con- 
taron con la presencia de un Médico Radiólogo en el grupo de trabajo.

Además, se obtuvo la proporción de discordancia entre la recomendación de las Guías Clínicas y la garantía de la prestación en cuanto a los exámenes imagenológicos.

Finalmente, se exploró si existió asociación entre la ausencia de Médico Radiólogo en el grupo de trabajo y la presencia de discordancia entre la recomendación de las Guías Clínicas GES y la garantía en el Listado de Prestaciones Específicas en cuanto a los exámenes imagenológicos. Para esto se realizó una tabla $2 \times 2$ y se realizó análisis mediante la prueba exacta de Fisher. La significancia se fijó en $p=0,05$ bilateral.

\section{Resultados Guías Clínicas GES}

Se encontraron 89 guías clínicas disponibles asociadas a las 80 patologías GES, 9 de éstas fueron subtemas de algunas de las 80 patologías y es por eso que se considera que constituyen una guía aparte, pero no una patología GES separada.

De las 89 guías analizadas, 60 contaban con recomendación de uso de exámenes imagenológicos y 29 no poseían recomendación de estos.

El total de exámenes imagenológicos recomendados fue de 135 clasificados en 10 modalidades de estudio: radiografía, ecotomografía, ecotomografía Doppler, tomografía axial computada (TAC), resonancia magnética (RM), tomografía de emisión de positrones con tomografía computada (PET-CT), angiografía, mamografía, evaluación ecográfica focalizada en trauma (ECOFAST) y estudio no especificado, con una mediana de 2 [IQR 1-3]. La modalidad de estudio más recomendada fue la radiografía simple.

\section{Listado de Prestaciones Específicas}

El listado de prestaciones específicas para las guías GES, cuenta con una "canasta de garantías" para las 80 guías clínicas que existían hasta el 2016. De las patologías presentadas, 55 contaban con exámenes imagenológicos en su listado de prestaciones garantizadas. Los exámenes imagenológicos incluidos fueron un total de 125, con una mediana de 2 [IQR 1-3]. La modalidad de estudio con mayor presencia en las canastas fue la radiografía simple, seguida del TAC (Figura 1).

En las 89 Guías se recomiendan en total 135 exámenes imagenológicos, mientras que en el Listado de Prestaciones Específicas se garantizan 125.

Se documentó la existencia de exámenes que eran recomendados en algunas Guías GES, pero o estaban en el Listado de Prestaciones Específicas respectivo y también, exámenes que, si bien estaban garantizados en el Listado de Prestaciones Específicas, no se encontraban con una recomendación explícita en la Guía GES correspondiente (4 guías,
Exámenes imagenológicos en Listado de Prestaciones Especificas según modalidad de estudio

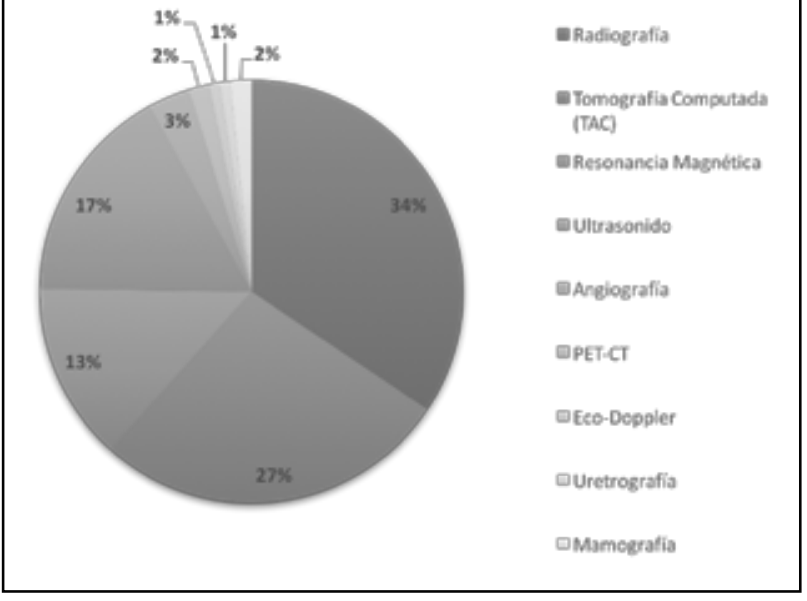

Figura 1: Porcentaje de exámenes en Listado de Prestaciones Específicas por modalidad de estudio.

8 exámenes). Se sumaron los 125 exámenes imagenológicos por modalidad garantizados en el Listado de Prestaciones Específicas con los recomendados en las Guías pero que no cuentan con la garantía correspondiente en el Listado de Prestaciones Específicas (7 guías, 14 exámenes), dando un total de 139 exámenes. Esta discrepancia fue observada en un $15,8 \%$ de los exámenes analizados.

Se documentó la presencia de Médico Radiólogo en 10 de las 89 Guías GES (Tabla 1). Observamos una discrepancia de $0 \%$ en los exámenes cuando hubo presencia de médico radiólogo en la Guía Clínica GES, mientras que se encontró un $19,3 \%$ de exámenes con discrepancia entre la recomendación y el listado de prestación cuando no hubo presencia del especialista en radiología. Esta diferencia fue estadísticamente significativa ( $p=0,007$, test exacto de Fisher).

Se calculó el costo de la discordancia con base en los aranceles Modalidad de Atención Institucional (MAI) 2019 ${ }^{(12)}$ para cada examen de cada guía o listado de prestación que presentaban discordancia, éstos se multiplicaron por los nuevos ingresos GES 2017(13) a cada patología con discordancia. El resultado fue de US\$27.387.917 equivalente a 9,1\% del Producto Interno Bruto (PIB) de 2018 de MUS\$298.231,14(14).

\section{Discusión}

Los exámenes imagenológicos son fundamentales en el diagnóstico y seguimiento de las diferentes enfermedades. Las guías clínicas GES y el Listado de Prestaciones Específicas dan un lugar muy importante a los exámenes imagenológicos, considerándolos en el $67 \%$ y $68 \%$, respectivamente. 
Tabla 1. Test exacto de Fisher, entre la presencia de Radiólogos y la discrepancia entre las Guías Clínicas GES y el Listado de Prestaciones Específicas.

\begin{tabular}{|l|cccc|}
\hline \multicolumn{5}{|c|}{ MÉDICO RADIÓLOGO } \\
\hline & & SI & NO & TOTAL \\
\hline \multirow{2}{*}{ DISCORDANCIA } & SI & 30 & 88 & 118 \\
& NO & 0 & 21 & 21 \\
& TOTAL & 30 & 109 & 139 \\
& & & & \\
\hline
\end{tabular}

Sin embargo, en sólo 10 Guías Clínicas GES de las 89 totales hubo participación de médicos radiólogos, por lo que es fundamental la ampliación de la participación de especialistas de esta área, ya que potencialmente mejoraría la utilización de los recursos, disminuiría los costos por exámenes innecesarios y reduciría la exposición de los pacientes a radiación ionizante médica innecesaria ${ }^{(15)}$.

Por otro lado, mientras que la realización de radiografías en el sistema de prestadores privados de salud aumentó en un $4 \%$, las tomografías computadas y las resonancias magnéticas lo hicieron en un 6\% y $15 \%$ respectivamente ${ }^{(9)}$. Esto da carácter prioritario a la participación de los Médicos Radiólogos en el desarrollo de las guías clínicas, debido al manejo que esta especialidad posee de exámenes imagenológicos de mayor complejidad, contribuyendo a darles el mejor uso posible.

Dada la asociación observada en la prueba exacta de Fisher, entre la ausencia de médico radiólogo y la mayor discrepancia entre las Guías Clínicas GES y el Listado de Prestaciones Específicas, se considera que la presencia de un médico radiólogo en la elaboración de estas podría ayudar a disminuir el grado de discrepancia.

Cabe destacar que existen guías norteamericanas y europeas con recomendaciones de exámenes imagenológicos para diferentes patologías que podrían ser consideradas a futuro en una actualización de las Guías GES, por lo que en países desarrollados es un problema resuelto. Sin embargo, estas guías no son desarrolladas a partir de políticas públicas como es el caso del GES, si no por sociedades médicas y en un contexto económico y social que difiere al de Chile. Por ello, sería de utilidad la elaboración de recomendaciones nacionales que sirvan de apoyo a la toma de decisiones para la utilización de exámenes imagenológicos, generadas por expertos nacionales, y considerando la realidad particular de nuestro país.

El costo asociado a las discrepancias en el caso del Listado de Prestaciones Específicas asciende a US\$2.887.221,16 y en el caso de las Guías GES US\$24.500.696,52. En el primer caso, al ser una prestación garantizada, pero no recomendada en la Guía GES correspondiente, por lo tanto, sin el argumento científico de por medio que lo avale, este es un costo que asume el Estado. En el segundo caso, son prestaciones que se encuentran recomendadas en las Guías GES, y que, si bien pueden existir diversos motivos por los que no se garantizan, tales como falta de equipos en todas las regiones del país para poder otorgar la prestación, el resultado final es que no están garantizadas. Esto implica un costo social y ético, ya que, en un Régimen de Garantías basado en la solidaridad y justicia social, las Guías presentan recomendaciones que, por diversos motivos, no se garantizan y que dado el carácter de ley que comprende el Régimen de Garantías Explícitas, estas prestaciones debieran estar aseguradas.

Una de las limitaciones de este trabajo es contar sólo con una aproximación teórica de la cantidad de exámenes imagenológicos que incluye cada una de las patologías contempladas en las Guías Clínicas GES y que se encuentran descritas en las canastas de prestaciones, sin embargo, no siempre coinciden con las recomendaciones de expertos. Esto se suma a la ausencia de bases de datos que indiquen el número real de estudios imagenológicos realizados durante las diferentes etapas de cada patología, lo que podría incluso superar lo garantizado en las guías, por lo que el rol de las imágenes podría estar subestimado.

De lo anterior se deduce que no es posible conocer la carga real de trabajo para los servicios de radiología y las consecuencias en la priorización de estudios GES por los no GES, dado que se debe cumplir con un plazo determinado.

Otra de las limitaciones es la posible connotación negativa que puede existir frente a la discordancia entre el listado de prestaciones garantizadas con protección económica y las que efectivamente se solicitan y que no necesariamente tendrán esta protección, ya que la mera existencia de una prestación garantizada podría tener una valoración social positiva.

Creemos que la participación de las distintas sociedades médicas en la elaboración de guías de práctica clínica, recomendaciones y políticas públicas 
que tienen impacto en la salud de la población, es fundamental para una mejor estandarización de la atención médica y optimización de recursos tanto fiscales como privados.

\section{Agradecimientos}

Al directorio de la Sociedad Chilena de Radiología (SOCHRADI) por su constante apoyo y promoción a las actividades académicas.

\section{Referencias}

1. Bastías G, Valdivia G. Reforma de salud en Chile; el plan AUGE o Régimen de Garantías Explicitas en Salud (GES). Su origen y evolución. Boletín Escuela de Medicina UC, Pontificia Universidad Católica de Chile. 2007; 32(2): 51-58.

2. Valdivieso V, Montero J. El plan AUGE: 2005 al 2009. Rev Med Chile. 2010; 138(8): 1040-1046.

3. Lenz, R. Proceso político de la Reforma AUGE de salud en Chile: Algunas lecciones para América Latina, una mirada desde la economía política. Serie de Estudios Socio económicos, CIEPLAN. 2007; 38.

4. Comunicados: Presidente Piñera aumenta cobertura de salud del Plan Auge incorporando cuatro tipos de cáncer y Alzheimer: "Queremos dar una respuesta a esos chilenos y chilenas que tienen que enfrentar la adversidad". Prensa Presidencia. Sitio Web: https:// prensa. presidencia. cl/comunicado. aspx $?$ id $=98130$ Updated julio 1, 2019. Consultado Julio 3, 2019.

5. Núñez P, Torres A, Armas R. ¿Se sustentan las Guías GES en trabajos científicos financiados por los fondos de CONICYT? Rev Med Chile. 2014; 142: 1540-1546.

6. Curso autogestionado GES. Sitio web http://capacitacion.siad-sps.cl/cursoges/modulos2/ modulo1/documentos.php. Consultado junio 5, 2019.
7. Amis $E$, Buttler P. ACR White paper on radiation dose in medicine: three years later. J Am Coll Radio. 2007; 4(5): 272-284.

8. MedPAC, A Data Book: Healthcare Spending and the Medicare Program. 2013. Sitio web: http://67.59.137.244/ documents/Jun13DataBookEntireReport.pdf Consultado junio 10, 2019.

9 Costos crecientes de la salud: Evolución y factores que están llevando a un mayor uso de las prestaciones médicas en el sistema de isapres. Isapres de Chile A.G. Serie de informes técnicos. 2015. Sitio web: http:// www.isapre.cl/PDF/Informe $\% 20$ Costos $\% 20$ de $\% 20$ Salud_Prestaciones\%20\%20(30\%2009\%202015)\%20 (5).pdf Consultado junio 7, 2019.

10. Guías Clínicas AUGE. Ministerio de Salud de Chile. Sitio Web: https://diprece.minsal .cl/le-informamos/auge/ acceso-guias-clinicas/guias-clinicas-auge/ Consultado abril 24, 2019.

11. Listado de Prestaciones Específicas. Ministerio de Salud de Chile. http://diprece.minsal.cl/wrdprss_minsal/ wp-content/uploads/2018/03/Lep_incluye-Decreto8-de-2018.pdf. Consultado mayo 16, 2019.

12. FONASA. Modalidad de Atención Institucional (MAI). https://www.fonasa.cl/sites/fonasa/prestadores/normativa/ aranceles\#modalidad-de-atencion-institucional--maiConsultado julio 21, 2019.

13. Garantías Explícitas Salud GES. Casos acumulados a junio de 2017. (Ingresos entre enero y diciembre 2017). http://www.supersalud.gob.cl/documentacion/666/w3propertyvalue-3746.html Consultado julio 21, 2019.

14. The World Bank. GDP (Current US $\$$ ) https://data. worldbank.org/indicator/NY.GDP.MKTP.CD? name desc=false14.- Consultado julio 21, 2019.

15 Arce J, García C, Moenne K, Bosch E. Uso innecesario de exámenes de imagen. Revista Chilena de Radiología. 2017; 23(3): 140-141. 